\title{
Buckling Analysis of Functionally Graded Material Plates Using Higher Order Shear Deformation Theory
}

\author{
B. Sidda Reddy, ${ }^{1}$ J. Suresh Kumar, ${ }^{2}$ C. Eswara Reddy, ${ }^{3}$ and K. Vijaya Kumar Reddy ${ }^{2}$ \\ ${ }^{1}$ School of Mechanical Engineering, R.G.M College of Engineering \& Technology, Nandyal, Kurnool, Andhra Pradesh 518 501, India \\ ${ }^{2}$ Department of Mechanical Engineering, J.N.T.U.H College of Engineering, J.N.T. University, Hyderabad, \\ Andhra Pradesh 500 085, India \\ ${ }^{3}$ The School of Engineering \& Technology, Sri Padmavathi Mahila Visvavidyalayam, Women's University, Tirupati, Chittoor, \\ Andhra Pradesh 517 502, India
}

Correspondence should be addressed to B. Sidda Reddy; bsrrgmcet@gmail.com

Received 29 May 2013; Revised 7 October 2013; Accepted 11 October 2013

Academic Editor: Serge Abrate

Copyright (C) 2013 B. Sidda Reddy et al. This is an open access article distributed under the Creative Commons Attribution License, which permits unrestricted use, distribution, and reproduction in any medium, provided the original work is properly cited.

\begin{abstract}
The prime aim of the present study is to present analytical formulations and solutions for the buckling analysis of simply supported functionally graded plates (FGPs) using higher order shear deformation theory (HSDT) without enforcing zero transverse shear stresses on the top and bottom surfaces of the plate. It does not require shear correction factors and transverse shear stresses vary parabolically across the thickness. Material properties of the plate are assumed to vary in the thickness direction according to a power law distribution in terms of the volume fractions of the constituents. The equations of motion and boundary conditions are derived using the principle of virtual work. Solutions are obtained for FGPs in closed-form using Navier's technique. Comparison studies are performed to verify the validity of the present results from which it can be concluded that the proposed theory is accurate and efficient in predicting the buckling behavior of functionally graded plates. The effect of side-to-thickness ratio, aspect ratio, modulus ratio, the volume fraction exponent, and the loading conditions on the critical buckling load of FGPs is also investigated and discussed.
\end{abstract}

\section{Introduction}

Functionally graded materials (FGMs) are the new generation of novel composite materials in the family of engineering composites, whose properties are varied smoothly in the spatial direction microscopically to improve the overall structural performance. These materials offer great promise in high temperature environments, for example, wear-resistant linings for handling large heavy abrasive ore particles, rocket heat shields, heat exchanger tubes, thermoelectric generators, heat engine components, plasma facings for fusion reactors, and electrically insulating metal/ceramic joints and also these are widely used in many structural applications such as mechanics, civil engineering, optical, electronic, chemical, mechanical, biomedical, energy sources, nuclear, automotive fields, and ship building industries to minimize thermomechanical mismatch in metal-ceramic bonding. Most structures, irrespective of their use, will be subjected to dynamic loads during their operational life. Increased use of FGMs in various structural applications necessitates the development of accurate theoretical models to predict their response.

In the past, a variety of plate theories have been proposed to study the buckling behavior of FGM plates. The classical plate theory $(\mathrm{CPT})$ provides acceptable results only for the analysis of thin plates and neglects the transverse shear effects. Javaheri and Eslami [1], Abrate [2], Mohammadi et al. [3], Mahdavian [4], Feldman and Aboudi [5], Shariat et al. [6], and Tung and Duc [7] employed this theory to analyze buckling behavior of FG plates. However, for moderately thick plates CPT underpredicts deflections and overpredicts buckling loads and natural frequencies. The first-order shear deformation theories (FSDTs) are based on Reissner [8] and Mindlin [9] accounts for the transverse shear deformation effect by means of a linear variation of inplane displacements and stresses through the thickness of the plate, 
but requires a correction factor to satisfy the free transverse shear stress conditions on the top and bottom surfaces of the plate. Although the FSDT provides a sufficiently accurate description of response for thin to moderately thick plates, it is not convenient to use due to difficulty with determination of the correct value of shear correction factor [10]. The authors [11-16] used FSDT to analyze the buckling of FG plates. In order to overcome the limitations of FSDT many HSDTs were developed that involve higher order terms in Taylors expansions of the displacements in the thickness coordinate. Javaheri and Eslami [17], Najafizadeh and Heydari [18], Bodaghi and Saidi [19], Bagherizadeh et al. [20], and Mozafari and Ayob [21] used the HSDT to analyze the buckling behavior of FG plates. Ma and Wang [22] have investigated the axisymmetric large deflection bending and postbuckling behavior of a functionally graded circular plate under mechanical, thermal, and combined thermal-mechanical load based on classical nonlinear von Karman plate theory. They observed from their investigation that the power law index " $n$ " has a significant effect on the midplane temperature, critical buckling temperature, and on the thermal post-buckling behavior of FGM plate. Hosseini-Hashemi et al. [23] have developed the closedform solutions in analytical form to study the buckling behavior of in-plane loaded isotropic rectangular FG plates without any use of approximation for different boundary conditions using the Mindlin plate theory. Saidi et al. [24] employed the unconstrained third-order shear deformation theory to analyze the axisymmetric bending and buckling of FG solid circular plates in which the bending-stretching coupling exists. Oyekoya et al. [25] developed Mindlin type and Ressner type element for modeling of FG composite plate subjected to buckling and free vibration. Further, they studied the plate for the effect of different fiber distribution cases and the effects of fire distribution on buckling, and free vibration. Ghannadpour et al. [26] applied finite strip method to analyze the buckling behavior of rectangular FG plats under thermal load. The solution was obtained by the minimization of the total potential energy and solving the corresponding eigenvalue problem. Thai and Choi [27] presented a simple refined theory to analyze the buckling behavior of FG plates which has strong similarity with classical plate theory in many aspects, accounts for a quadratic variation of the transverse shear strains across the thickness, and satisfies the zero traction boundary conditions on the top and bottom surfaces of the plate without using shear correction factors. The governing equations were derived from the principle of minimum total potential energy. The effects of loading conditions and variations of power of functionally graded material, modulus ratio, aspect ratio, and thickness ratio were also investigated by these authors. Thai and Vo [10] have developed a new sinusoidal shear deformation theory to study the bending, buckling and vibration of FG plates accounting for sinusoidal distribution of transverse shear stress and satisfies the free transverse shear stress conditions on the top and bottom surfaces of the plate without using shear correction factor. Uymaz and Aydogdu [28] analyzed the rectangular FG plates under different axial loadings for buckling based on small strain elasticity theory with different boundary conditions. They also investigated the effects of the different material composition and the plate geometry on the critical buckling loads and mode shapes.

Lal et al. [29] have examined the second order statistics of postbuckling responses of FGM plate subjected to mechanical and thermal load with nonuniform temperature changes subjected to temperature independent and dependent material properties. The effect of random material properties with amplitude ratios, volume fraction index, plate thickness ratios, aspect ratios, boundary conditions, and types of loadings subjected to temperature independent and temperature dependent material properties were investigated through numerical examples.

This paper aims to develop analytical formulations and solutions for the buckling analysis of functionally graded plates (FGPs) using higher order shear deformation theory (HSDT) without enforcing zero transverse shear stress on the top and bottom surfaces of the plate. This does not require shear correction factor. The plate material is graded through the thickness direction. The plate's governing equations and its boundary conditions are derived by employing the principle of virtual work. Solutions are obtained for FGPs in closed-form using Navier's technique and solving the eigenvalue equation. The present results are compared with the solutions of Thai and Choi [27] to verify the accuracy of the proposed theory in predicting the critical buckling loads of FG plates. The effect of side-to-thickness ratios, aspect ratios, and modulus ratios and the volume fraction exponent on the critical buckling loads are studied after establishing the accuracy of the present results for FG plates.

\section{Theoretical Formulation}

In formulating the higher order shear deformation theory, a rectangular plate of length $a$, width $b$, and thickness $h$ is considered, which composed of functionally graded material through the thickness. Figure 1 shows the functionally graded material plate with the rectangular Cartesian coordinate system $x, y$, and $z$. The material properties are assumed to be varied in the thickness direction only and the bright and dark areas correspond to ceramic and metal particles, respectively. On the top surface $(z=+h / 2)$, the plate is composed of full ceramic and graded to the bottom surface $(z=-h / 2)$ which composed of full metal. The reference surface is the middle surface of the plate $(z=0)$. The functionally graded material plate properties are assumed to be the function of the volume fraction of constituent materials. The functional relationship between the material property and the thickness coordinates is assumed to be

$$
P(z)=\left(P_{t}-P_{b}\right)\left(\frac{z}{h}+\frac{1}{2}\right)^{n}+P_{b}
$$

where $P$ denotes the effective material property, $P_{t}$ and $P_{b}$ denote the property on the top and bottom surface of the plate, respectively, and $n$ is the material variation parameter that dictates the material variation profile through the thickness. The effective material properties of the plate, including Young's modulus, $E$, density, $\rho$, and shear modulus, 


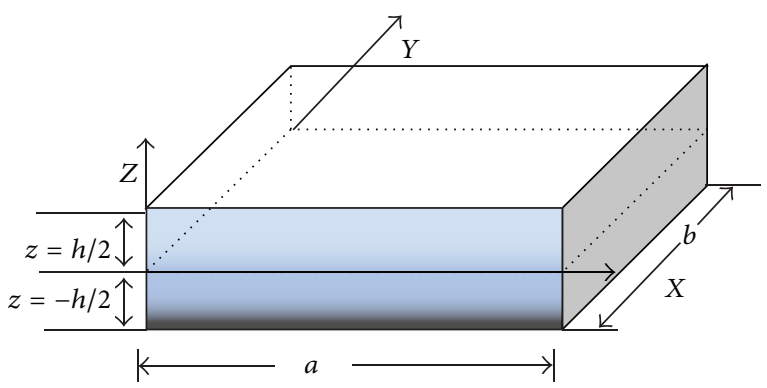

FIGURE 1: Functionally graded plate and coordinates.

$G$, vary according to (1), and poisons ratio $(v)$ is assumed to be constant.

2.1. Displacement Models. In order to approximate 3D plate problem to a $2 \mathrm{D}$ one, the displacement components $u(x, y, z, t), v(x, y, z, t)$, and $w(x, y, z, t)$ at any point in the plate are expanded in terms of the thickness coordinate. The elasticity solution indicates that the transverse shear stress varies parabolically through the plate thickness. This requires the use of a displacement field, in which the inplane displacements are expanded as cubic functions of the thickness coordinate. In addition, the transverse normal strain may vary nonlinearly through the plate thickness. The displacement field which satisfies the above criteria may be assumed in the form:

$$
\begin{aligned}
u(x, y, z)= & u_{o}(x, y)+z \theta_{x}(x, y) \\
& +z^{2} u_{o}^{*}(x, y)+z^{3} \theta_{x}^{*}(x, y) \\
v(x, y, z)= & v_{o}(x, y)+z \theta_{y}(x, y) \\
& +z^{2} v_{o}^{*}(x, y)+z^{3} \theta_{y}^{*}(x, y) \\
w(x, y, z)= & w_{o}(x, y),
\end{aligned}
$$

where $u_{o}, v_{o}$, and $w_{o}$ denote the displacements of a point $(x, y)$ on the midplane.

$\theta_{x}, \theta_{y}$ are rotations of the normal to the mid plane about $y$ and $x$-axes.

$u_{0}^{*}, v_{0}^{*}, \theta_{x}^{*}$, and $\theta_{y}^{*}$ are the higher order deformation terms defined at the mid plane.

By substitution of displacement relations from (2) into the strain displacement equations of the classical theory of elasticity, the following relations are obtained:

$$
\begin{gathered}
\varepsilon_{x}=\varepsilon_{x o}+z k_{x}+z^{2} \varepsilon_{x o}^{*}+z^{3} k_{x}^{*}, \\
\varepsilon_{y}=\varepsilon_{y o}+z k_{y}+z^{2} \varepsilon_{y o}^{*}+z^{3} k_{y}^{*}, \\
\varepsilon_{z}=0, \\
\gamma_{x y}=\varepsilon_{x y o}+z k_{x y}+z^{2} \varepsilon_{x y o}^{*}+z^{3} k_{x y}^{*}, \\
\gamma_{y z}=\varphi_{y}+z \varepsilon_{y z o}+z^{2} \varphi_{y}^{*}, \\
\gamma_{x z}=\varphi_{x}+z \varepsilon_{x z o}+z^{2} \varphi_{x}^{*},
\end{gathered}
$$

where

$$
\begin{gathered}
\varepsilon_{x o}=\frac{\partial u_{o}}{\partial x}, \quad \varepsilon_{y o}=\frac{\partial v_{o}}{\partial y}, \quad \varepsilon_{x y o}=\frac{\partial u_{o}}{\partial y}+\frac{\partial v_{o}}{\partial x} \\
k_{x}=\frac{\partial \theta_{x}}{\partial x}, \quad k_{y}=\frac{\partial \theta_{y}}{\partial y}, \quad k_{x y}=\frac{\partial \theta_{x}}{\partial y}+\frac{\partial \theta_{y}}{\partial x} \\
k_{x}^{*}=\frac{\partial \theta_{x}^{*}}{\partial x}, \quad k_{y}^{*}=\frac{\partial \theta_{y}^{*}}{\partial y}, \quad k_{x y}^{*}=\frac{\partial \theta_{x}^{*}}{\partial y}+\frac{\partial \theta_{y}^{*}}{\partial x} \\
\varepsilon_{x o}^{*}=\frac{\partial u_{o}^{*}}{\partial x}, \quad \varepsilon_{y o}^{*}=\frac{\partial v_{o}^{*}}{\partial y}, \quad \varepsilon_{x y o}^{*}=\frac{\partial u_{o}^{*}}{\partial y}+\frac{\partial v_{o}^{*}}{\partial x} \\
\varepsilon_{y z o}=2 v_{o}^{*}, \quad \varepsilon_{x z o}=2 u_{o}^{*}, \quad \varphi_{y}^{*}=3 \theta_{y}^{*}, \quad \varphi_{x}^{*}=3 \theta_{x}^{*} .
\end{gathered}
$$

2.2. Elastic Stress-Strain Relations. The elastic stress-strain relations depend on which assumption of $\varepsilon_{z}=0$. In the case of functionally graded materials the constitutive equations can be written as

$$
\left\{\begin{array}{c}
\sigma_{x} \\
\sigma_{y} \\
\tau_{x y} \\
\tau_{y z} \\
\tau_{x z}
\end{array}\right\}=\left[\begin{array}{ccccc}
Q_{11} & Q_{12} & 0 & 0 & 0 \\
Q_{12} & Q_{22} & 0 & 0 & 0 \\
0 & 0 & Q_{33} & 0 & 0 \\
0 & 0 & 0 & Q_{44} & 0 \\
0 & 0 & 0 & 0 & Q_{55}
\end{array}\right]\left[\begin{array}{c}
\varepsilon_{x} \\
\varepsilon_{y} \\
\gamma_{x y} \\
\gamma_{y z} \\
\gamma_{x z}
\end{array}\right],
$$

where $\sigma=\left(\sigma_{x}, \sigma_{y}, \tau_{x y}, \tau_{y z}, \tau_{x z}\right)^{t}$ are the stresses, $\varepsilon=$ $\left(\varepsilon_{x}, \varepsilon_{y}, \gamma_{x y}, \gamma_{y z}, \gamma_{x z}\right)^{t}$ are the strains with respect to the axes, and $Q_{i j}$ 's are the plane stress reduced elastic coefficients in the plate axes that vary through the plate thickness given by

$$
\begin{gathered}
Q_{11}=Q_{22}=\frac{E(Z)}{1-v^{2}} \\
=\frac{\left(E_{c}-E_{m}\right)((z / h)+(1 / 2))^{n}+E_{m}}{1-v^{2}}, \\
Q_{12}=Q_{21}=v Q_{11}, \\
Q_{33}=Q_{44}=Q_{55}=\frac{\left(1-v^{2}\right)}{2(1+v)} Q_{11},
\end{gathered}
$$

where $E_{c}$ is the modulus of elasticity of the ceramic material and $E_{m}$ is the modulus of elasticity of the metal.

2.3. Governing Equations of Motion. The work done by the actual forces in moving through virtual displacements, which are consistent with the geometric constraints of a body, is set to zero to obtain the equation of motion and this is known as energy principle. It is useful in (a) deriving governing equations and the boundary conditions and (b) obtaining approximate solutions by virtual methods.

Energy principles provide alternative means to obtain the governing equations and their solutions. In the present study, 
the principle of virtual work is used to derive the equations of motion of functionally graded plates.

The governing equations of displacement model in (2) will be derived using the dynamic version of the principle of virtual displacements; that is,

$$
\int_{0}^{T}(\delta U+\delta V-\delta K) d t=0
$$

where $\delta U=$ virtual strain energy, $\delta V=$ virtual work done by applied forces, $\delta K=$ virtual kinetic energy, $\delta U+\delta V=$ total potential energy.

The virtual strain energy, work done, and kinetic energy is given by

$$
\begin{gathered}
\delta U=\int_{A}\left\{\int _ { - h / 2 } ^ { h / 2 } \left[\sigma_{x} \delta \epsilon_{x}+\sigma_{y} \delta \epsilon_{y}+\tau_{x y} \delta \gamma_{x y}\right.\right. \\
\left.\left.+\tau_{x z} \delta \gamma_{x z}+\tau_{y z} \delta \gamma_{y z}\right] d z\right\} d x d y \\
\delta V=-\int\left(q w_{0}+\bar{N}_{x} \frac{\partial w_{o}}{\partial x} \frac{\delta \partial w_{o}}{\partial x}+\bar{N}_{x y} \frac{\partial w_{o}}{\partial y} \frac{\delta \partial w_{o}}{\partial x}\right. \\
\left.+\bar{N}_{y x} \frac{\partial w_{o}}{\partial x} \frac{\delta \partial w_{o}}{\partial y}+\bar{N}_{y} \frac{\partial w_{o}}{\partial y} \frac{\delta \partial w_{o}}{\partial y}\right) d x d y \\
\int_{A}\left\{\int _ { - h / 2 } ^ { h / 2 } \rho _ { 0 } \left[\left(\dot{u}_{0}+z \dot{\theta}_{x}+z^{2} \dot{u}_{0}^{*}+z^{3} \dot{\theta}_{x}^{*}\right)\right.\right. \\
\times\left(\delta \dot{u}_{0}+z \delta \dot{\theta}_{x}+z^{2} \delta \dot{u}_{0}^{*}+z^{3} \delta \dot{\theta}_{x}^{*}\right) \\
+\left(\dot{v}_{0}+z \dot{\theta}_{y}+z^{2} \dot{v}_{0}^{*}+z^{3} \dot{\theta}_{y}^{*}\right) \\
\times\left(\delta \dot{v}_{0}+z \delta \dot{\theta}_{y}+z^{2} \delta \dot{v}_{0}^{*}+z^{3} \delta \dot{\theta}_{y}^{*}\right) \\
\left.\left.+\dot{w}_{0} \delta \dot{w}_{0}\right] d z\right\} d x d y
\end{gathered}
$$

where $q=$ distributed load over the surface of the plate.

$\bar{N}_{x}$ and $\bar{N}_{y}$ the inplane loads perpendicular to the edges $x=0$ and $y=0$, respectively, $\bar{N}_{x y} \bar{N}_{y x}$ the distributed shear forces parallel to the edges $x=0$ and $y=0$, respectively, $\rho_{0}=$ density of plate material, $\dot{u}_{0}=\partial u 0 / \partial t, \dot{v}_{0}=\partial v 0 / \partial t$, and so forth, indicate the time derivatives.

Substituting for $\delta U, \delta V$, and $\delta K$ in the virtual work statement in (7) and integrating through the thickness, integrating by parts, and collecting the coefficients of $\delta u_{o}, \delta v_{o}, \delta w_{o}, \delta \theta_{x}, \delta \theta_{y}, \delta u_{o}^{*}, \delta v_{o}^{*}, \delta \theta_{x}^{*}, \delta \theta_{y}^{*}$, the following equations of motion are obtained:

$$
\begin{aligned}
\delta u_{0}: & \frac{\partial N_{x}}{\partial x}+\frac{\partial N_{x y}}{\partial y} \\
& =I_{1} \ddot{u}_{0}+I_{2} \ddot{\theta}_{x}+I_{3} \ddot{u}_{0}^{*}+I_{4} \ddot{\theta}_{x}^{*},
\end{aligned}
$$

$$
\begin{gathered}
\delta v_{0}: \frac{\partial N_{y}}{\partial y}+\frac{\partial N_{x y}}{\partial x} \\
=I_{1} \ddot{v}_{0}+I_{2} \ddot{\theta}_{y}+I_{3} \ddot{v}_{0}^{*}+I_{4} \ddot{\theta}_{y}^{*}, \\
\delta w_{0}: \frac{\partial Q_{x}}{\partial x}+\frac{\partial Q_{y}}{\partial y}+q+\widetilde{N}=I_{1} \ddot{w}_{0},
\end{gathered}
$$

where

$$
\begin{aligned}
\widetilde{N}=\bar{N}_{x} \frac{\partial^{2} w_{o}}{\partial x^{2}}+\bar{N}_{x y} \frac{\partial^{2} w_{o}}{\partial y \partial x}+\bar{N}_{y x} \frac{\partial^{2} w_{o}}{\partial x \partial y}+\bar{N}_{y} \frac{\partial^{2} w_{o}}{\partial y^{2}}, \\
\delta \theta_{x}: \frac{\partial M_{x}}{\partial x}+\frac{\partial M_{x y}}{\partial y}-Q_{x} \\
=I_{2} \ddot{u}_{0}+I_{3} \ddot{\theta}_{x}+I_{4} \ddot{u}_{0}^{*}+I_{5} \ddot{\theta}_{x}^{*}, \\
\delta \theta_{y}: \frac{\partial M_{y}}{\partial y}+\frac{\partial M_{x y}}{\partial x}-Q_{y} \\
=I_{2} \ddot{v}_{0}+I_{3} \ddot{\theta}_{y}+I_{4} \ddot{v}_{0}^{*}+I_{5} \ddot{\theta}_{y}^{*}, \\
\delta u_{0}^{*}: \frac{\partial N_{x}^{*}}{\partial x}+\frac{\partial N_{x y}^{*}}{\partial y}-2 S_{x} \\
\delta \theta_{y}^{*}: \frac{\partial M_{y}^{*}}{\partial y}+\frac{\partial M_{x y}^{*}}{\partial x}-3 Q_{y} \\
=I_{3} \ddot{u}_{0}+I_{4} \ddot{\theta}_{x}+I_{5} \ddot{u}_{0}^{*}+I_{6} \ddot{\theta}_{x}^{*}, \\
\delta \theta_{x}^{*}: \frac{\partial M_{x}^{*}}{\partial x}+\frac{\partial M_{x y}^{*}}{\partial y}+3 Q_{x}^{*} \\
=I_{4} \ddot{u}_{0}+I_{5} \ddot{\theta}_{x}+I_{6} \ddot{u}_{0}^{*}+I_{7} \ddot{\theta}_{x}^{*}, \\
=I_{3} \ddot{\theta}_{y}+I_{6} \ddot{v}_{0}^{*}+I_{7} \ddot{\theta}_{y}^{*}, \\
\\
: I_{4} \ddot{\theta}_{y}+I_{5} \ddot{v}_{0}^{*}+I_{6} \ddot{\theta}_{y}^{*},
\end{aligned}
$$

where the force and moment resultants are defined as

$$
\begin{aligned}
& \left\{\begin{array}{l|l}
N_{x} & N_{x}^{*} \\
N_{y} & N_{y}^{*} \\
N_{x y} & N_{x y}^{*}
\end{array}\right\}=\sum_{L=1}^{n} \int_{-h / 2}^{h / 2}\left\{\begin{array}{c}
\sigma_{x} \\
\sigma_{y} \\
\tau_{x y}
\end{array}\right\}\left[\begin{array}{lll}
1 & \mid & z^{2}
\end{array}\right] d z, \\
& \left\{\begin{array}{l|l}
M_{x} & M_{x}^{*} \\
M_{y} & M_{y}^{*} \\
M_{x y} & M_{x y}^{*}
\end{array}\right\}=\sum_{L=1}^{n} \int_{-h / 2}^{h / 2}\left\{\begin{array}{c}
\sigma_{x} \\
\sigma_{y} \\
\tau_{x y}
\end{array}\right\}\left[\begin{array}{lll}
z & \mid & z^{3}
\end{array}\right] d z .
\end{aligned}
$$


And the transverse force resultants and the inertias are given by

$$
\begin{aligned}
& \left\{\begin{array}{llll}
Q_{x} & \mid S_{x} & Q_{x}^{*} \\
Q_{y} & \mid & S_{y} & Q_{y}^{*}
\end{array}\right\} \\
& =\sum_{L=1}^{n} \int_{-h / 2}^{h / 2}\left\{\begin{array}{l}
\tau_{x z} \\
\tau_{y z}
\end{array}\right\}\left[\begin{array}{llll}
1 & \mid & z & z^{2}
\end{array}\right] d z, \\
& I_{1}, I_{2}, I_{3}, I_{4}, I_{5}, I_{6}, I_{7} \\
& =\int_{-h / 2}^{h / 2}\left[\left(\rho_{c}-\rho_{m}\right)\left(\frac{2 z-h}{2 h}\right)^{n}+\rho_{m}\right] \\
& \quad \times\left(1, z, z^{2}, z^{3}, z^{4}, z^{5}, z^{6}\right) d z .
\end{aligned}
$$

The resultants in (11)-(12) can be related to the total strains in (5) by the following matrix:

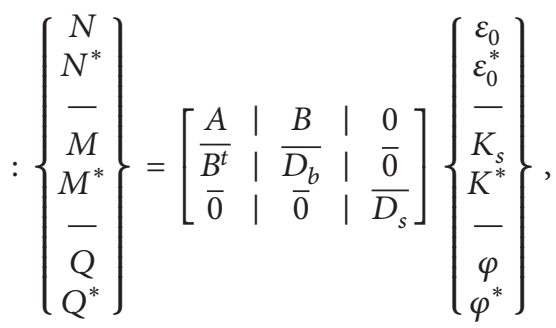

where

$$
N=\left[\begin{array}{lll}
N_{x} & N_{y} & N_{x y}
\end{array}\right]^{t ;} \quad N^{*}=\left[\begin{array}{lll}
N_{x}^{*} & N_{y}^{*} & N_{x y}^{*}
\end{array}\right]^{t} .
$$

$N, N^{*}$ are called the inplane force resultants

$$
M=\left[\begin{array}{lll}
M_{x} & M_{y} & M_{x y}
\end{array}\right]^{t} ; \quad M^{*}=\left[\begin{array}{lll}
M_{x}^{*} & M_{y}^{*} & M_{x y}^{*}
\end{array}\right]^{t} .
$$

$M, M^{*}$ are called as moment resultants

$$
Q=\left[\begin{array}{ll}
Q_{x} & Q_{y}
\end{array}\right]^{t} ; \quad Q^{*}=\left[\begin{array}{llll}
S_{x} & S_{y} & Q_{x}^{*} & Q_{y}^{*}
\end{array}\right]^{t}
$$

$Q, Q^{*}$ denote the transverse force result

$$
\begin{aligned}
\varepsilon_{0} & =\left[\begin{array}{lll}
\varepsilon_{x 0} & \varepsilon_{y 0} & \varepsilon_{x y 0}
\end{array}\right]^{t} ; \quad \varepsilon_{0}^{*}=\left[\begin{array}{lll}
\varepsilon_{x 0}^{*} & \varepsilon_{y 0}^{*} & \varepsilon_{x y 0}^{*}
\end{array}\right]^{t} \\
K_{s} & =\left[\begin{array}{lll}
K_{x} & K_{y} & K_{x y}
\end{array}\right]^{t} ; \quad K^{*}=\left[\begin{array}{lll}
K_{x}^{*} & K_{y}^{*} & K_{x y}^{*}
\end{array}\right]^{t} \\
\varphi & =\left[\begin{array}{ll}
\varphi_{x} & \varphi_{y}
\end{array}\right]^{t} ; \quad \varphi^{*}=\left[\begin{array}{llll}
\varepsilon_{x z 0} & \varepsilon_{y z 0} & \varphi_{x}^{*} & \varphi_{y}^{*}
\end{array}\right]^{t} .
\end{aligned}
$$

The matrices $[A],[B],[D]$, and $\left[D_{s}\right]$ are the plate stiffness whose elements can be calculated using (5) and (11)-(12).

\section{Analytical Solution for the Simply Supported Plate}

Let $a$ be simply supported rectangular plate with length $a$ and width $b$ which is subjected to in-plane loading in two directions $\left(\bar{N}_{x}=-\lambda_{1} N_{\mathrm{cr}}, \bar{N}_{y}=-\lambda_{2} N_{\mathrm{cr}}, \bar{N}_{x y}=\right.$ $0)$. The following expressions of displacements are chosen based on Navier's approach to automatically satisfy the simply supported boundary conditions of the plate:

$$
\begin{aligned}
u_{0}(x, y, t) & =\sum_{m=1}^{\infty} \sum_{n=1}^{\infty} U_{m n} \cos \alpha x \sin \beta y, \\
v_{0}(x, y, t) & =\sum_{m=1}^{\infty} \sum_{n=1}^{\infty} V_{m n} \sin \alpha x \cos \beta y, \\
w_{0}(x, y, t) & =\sum_{m=1}^{\infty} \sum_{n=1}^{\infty} W_{m n} \sin \alpha x \sin \beta y, \\
\theta_{x}(x, y, t) & =\sum_{m=1}^{\infty} \sum_{n=1}^{\infty} X_{m n} \cos \alpha x \sin \beta y, \\
\theta_{y}(x, y, t) & =\sum_{m=1}^{\infty} \sum_{n=1}^{\infty} Y_{m n} \sin \alpha x \cos \beta y, \\
u_{0}^{*}(x, y, t) & =\sum_{m=1}^{\infty} \sum_{n=1}^{\infty} U_{m n}^{*} \cos \alpha x \sin \beta y, \\
V_{o}^{*}(x, y, t)= & \sum_{m=1}^{\infty} \sum_{n=1}^{\infty} V_{m n}^{*} \sin \alpha x \cos \beta y, \\
\theta_{x}^{*}(x, y, t)= & \sum_{m=1}^{\infty} \sum_{n=1}^{\infty} X_{m n}^{*} \cos \alpha x \sin \beta y, \\
\theta_{y}(x, y, t) & \sum_{m=1}^{\infty} \sum_{n=1}^{\infty} Y_{m n}^{*} \sin \alpha x \cos \beta y .
\end{aligned}
$$

The eigenproblem related to governing equations is defined as

$$
\left([S]_{9 \times 9}-\lambda[\varsigma]_{9 \times 9}\right) \mathbf{X}=0,
$$

where $[S]$ collects all stiffness terms and $[\varsigma]$ collects all terms related to the in-plane forces. In (20) $\mathbf{X}$ are the modes of buckling associated with the buckling loads defined as $\lambda$. For each value of $m$ and $n$, there is a unique value of $N_{\mathrm{cr}}$. The critical buckling load is the smallest value of $N_{\mathrm{cr}}(m, n)$.

\section{Results and Discussion}

4.1. Comparative Studies. To validate the accuracy of the present higher order theory in predicting the critical buckling load of FG plates subjected to different in-plane loading conditions (uniaxial compression: $\lambda_{1}=-1, \lambda_{2}=0$; biaxial compression: $\lambda_{1}=-1, \lambda_{2}=-1$; biaxial compression and tension $\lambda_{1}=-1, \lambda_{2}=1$ ), four numerical examples are presented and discussed. The material properties adopted here are as follows.

Aluminium Young's modulus $\left(E_{m}\right): 70 \mathrm{GPa}$, density $\rho_{m}=$ $2702 \mathrm{~kg} / \mathrm{m}^{3}$, and Poisson's ratio $(v): 0.3$.

Alumina Young's modulus $\left(E_{c}\right): 380 \mathrm{GPa}$, density $\rho_{c}=$ $3800 \mathrm{~kg} / \mathrm{m}^{3}$, and Poisson's ratio $(v): 0.3$. 
TABLE 1: Comparison of nondimensionalized critical buckling load $(\bar{N})$ of simply supported $\mathrm{Al} / \mathrm{Al}_{2} \mathrm{O}_{3}$ plate subjected to uniaxial compression along the $x$-axis $\left(\lambda_{1}=-1, \lambda_{2}=0\right)$.

\begin{tabular}{|c|c|c|c|c|c|c|c|c|c|c|}
\hline \multicolumn{11}{|c|}{ Power-law index, $n$} \\
\hline$a / b$ & $a / h$ & Source & 0 & 0.5 & 1 & 2 & 5 & 10 & 20 & 100 \\
\hline \multirow{10}{*}{0.5} & \multirow{2}{*}{5} & {$[27]$} & 6.7203 & 4.4235 & 3.4164 & 2.6451 & 2.1484 & 1.9213 & 1.7115 & 1.3737 \\
\hline & & Present & 6.714 & 4.409 & 3.39 & 2.61 & 2.124 & 1.90 & 1.705 & 1.371 \\
\hline & \multirow{2}{*}{10} & {$[27]$} & 7.405 & 4.82 & 3.71 & 2.88 & 2.41 & 2.18 & 1.93 & 1.52 \\
\hline & & Present & 7.397 & 4.81 & 3.70 & 2.87 & 2.40 & 2.18 & 1.93 & 1.5231 \\
\hline & \multirow{2}{*}{20} & {$[27]$} & 7.599 & 4.93 & 3.79 & 2.95 & 2.49 & 2.26 & 2.00 & 1.56 \\
\hline & & Present & 7.590 & 4.924 & 3.78 & 2.95 & 2.48 & 2.26 & 2.00 & 1.56 \\
\hline & \multirow{2}{*}{50} & {$[27]$} & 7.65 & 4.96 & 3.81 & 2.97 & 2.51 & 2.29 & 2.025 & 1.58 \\
\hline & & Present & 7.64 & 4.95 & 3.81 & 2.973 & 2.51 & 2.28 & 2.02 & 1.57 \\
\hline & \multirow{2}{*}{100} & {$[27]$} & 7.66 & 4.968 & 3.82 & 2.98 & 2.52 & 2.29 & 2.028 & 1.58 \\
\hline & & Present & 7.65 & 4.96 & 3.81 & 2.97 & 2.51 & 2.292 & 2.02 & 1.58 \\
\hline \multirow{10}{*}{1} & \multirow{2}{*}{5} & [27] & 16.02 & 10.62 & 8.22 & 6.34 & 5.05 & 4.48 & 4.00 & 3.25 \\
\hline & & Present & 16.00 & 10.57 & 8.146 & 6.23 & 4.97 & 4.44 & 3.98 & 3.25 \\
\hline & \multirow{2}{*}{10} & [27] & 18.57 & 12.12 & 9.33 & 7.26 & 6.03 & 5.45 & 4.83 & 3.81 \\
\hline & & Present & 18.54 & 12.08 & 9.299 & 7.21 & 5.99 & 5.42 & 4.82 & 3.81 \\
\hline & \multirow{2}{*}{20} & [27] & 19.35 & 12.56 & 9.66 & 7.53 & 6.34 & 5.76 & 5.09 & 3.99 \\
\hline & & Present & 19.31 & 12.53 & 9.649 & 7.51 & 6.32 & 5.75 & 5.08 & 3.98 \\
\hline & \multirow{2}{*}{50} & [27] & 19.58 & 12.69 & 9.763 & 7.61 & 6.43 & 5.8 & 5.17 & 4.04 \\
\hline & & Present & 19.54 & 12.67 & 9.743 & 7.601 & 6.42 & 5.84 & 5.16 & 4.03 \\
\hline & \multirow{2}{*}{100} & [27] & 19.61 & 12.71 & 9.77 & 7.62 & 6.45 & 5.87 & 5.18 & 4.05 \\
\hline & & Present & 19.57 & 12.69 & 9.75 & 7.61 & 6.43 & 5.86 & 5.17 & 4.04 \\
\hline \multirow{10}{*}{1.5} & \multirow{2}{*}{5} & {$[27]$} & $28.19^{\mathrm{a}}$ & $19.25^{\mathrm{a}}$ & $15.03^{\mathrm{a}}$ & $7.61^{\mathrm{a}}$ & $8.47^{\mathrm{a}}$ & $7.29^{a}$ & $6.61^{a}$ & $5.63^{\mathrm{a}}$ \\
\hline & & Present & $28.15^{\mathrm{a}}$ & $19.09^{\mathrm{a}}$ & $14.76^{\mathrm{a}}$ & 11.06 & $8.25^{\mathrm{a}}$ & $7.20^{\mathrm{a}}$ & $6.56^{\mathrm{a}}$ & $5.612^{\mathrm{a}}$ \\
\hline & \multirow{2}{*}{10} & {$[27]$} & $40.74^{\mathrm{a}}$ & $26.90^{\mathrm{a}}$ & $20.80^{\mathrm{a}}$ & $16.07^{\mathrm{a}}$ & $12.95^{\mathrm{a}}$ & $11.53^{\mathrm{a}}$ & $10.29^{\mathrm{a}}$ & $8.31^{\mathrm{a}}$ \\
\hline & & Present & $40.58^{\mathrm{a}}$ & $26.72^{\mathrm{a}}$ & $20.57^{\mathrm{a}}$ & $15.81^{\mathrm{a}}$ & $12.74^{\mathrm{a}}$ & $11.42^{\mathrm{a}}$ & $10.22^{\mathrm{a}}$ & $8.27^{\mathrm{a}}$ \\
\hline & \multirow{2}{*}{20} & [27] & $45.89^{\mathrm{a}}$ & $29.90^{\mathrm{a}}$ & $23.02^{\mathrm{a}}$ & $17.92^{\mathrm{a}}$ & $14.94^{\mathrm{a}}$ & $13.52^{\mathrm{a}}$ & $11.98^{\mathrm{a}}$ & $9.44^{\mathrm{a}}$ \\
\hline & & Present & $45.64^{\mathrm{a}}$ & $29.71^{\mathrm{a}}$ & $22.85^{\mathrm{a}}$ & $17.75^{\mathrm{a}}$ & $14.81^{\mathrm{a}}$ & $13.425^{\mathrm{a}}$ & $11.90^{\mathrm{a}}$ & $9.39^{\mathrm{a}}$ \\
\hline & \multirow{2}{*}{50} & [27] & $47.57^{\mathrm{a}}$ & $30.86^{\mathrm{a}}$ & $23.74^{\mathrm{a}}$ & $18.51^{\mathrm{a}}$ & $15.628^{\mathrm{a}}$ & $14.21^{\mathrm{a}}$ & $12.56^{\mathrm{a}}$ & $9.82^{\mathrm{a}}$ \\
\hline & & Present & $47.29^{\mathrm{a}}$ & $30.67^{\mathrm{a}}$ & $23.59^{\mathrm{a}}$ & $18.39^{\mathrm{a}}$ & $15.51^{\mathrm{a}}$ & $14.12^{\mathrm{a}}$ & $12.48^{\mathrm{a}}$ & $9.76^{\mathrm{a}}$ \\
\hline & \multirow{2}{*}{100} & [27] & $47.82^{\mathrm{a}}$ & $31.01^{\mathrm{a}}$ & $23.84^{\mathrm{a}}$ & $18.60^{\mathrm{a}}$ & $15.72^{\mathrm{a}}$ & $14.31^{\mathrm{a}}$ & $12.65^{\mathrm{a}}$ & $9.87^{\mathrm{a}}$ \\
\hline & & Present & $47.53^{\mathrm{a}}$ & $30.82^{\mathrm{a}}$ & $23.69^{\mathrm{a}}$ & $18.48^{\mathrm{a}}$ & $15.62^{\mathrm{a}}$ & $14.23^{\mathrm{a}}$ & $12.57^{\mathrm{a}}$ & $9.81^{\mathrm{a}}$ \\
\hline \multirow{10}{*}{2} & \multirow{2}{*}{5} & {$[27]$} & $37.74^{\mathrm{b}}$ & $26.36^{\mathrm{b}}$ & $20.74^{\mathrm{b}}$ & $15.58^{\mathrm{b}}$ & $10.95^{\mathrm{b}}$ & $9.15^{c}$ & $8.39^{c}$ & $7.44^{\mathrm{b}}$ \\
\hline & & Present & $37.67^{\mathrm{b}}$ & $26.11^{\mathrm{b}}$ & $20.29^{\mathrm{b}}$ & $14.99^{\mathrm{b}}$ & $10.65^{\mathrm{b}}$ & $9.04^{c}$ & $8.317^{\mathrm{c}}$ & $7.40^{\mathrm{b}}$ \\
\hline & \multirow{2}{*}{10} & [27] & $64.08^{\mathrm{a}}$ & $42.50^{\mathrm{a}}$ & $32.89^{\mathrm{a}}$ & $25.37^{\mathrm{a}}$ & $20.21^{\mathrm{a}}$ & $17.92^{\mathrm{a}}$ & $16.02^{\mathrm{a}}$ & $13.03^{\mathrm{a}}$ \\
\hline & & Present & $63.78^{\mathrm{a}}$ & $42.14^{\mathrm{a}}$ & $32.46^{\mathrm{a}}$ & $24.86^{\mathrm{a}}$ & $19.84^{\mathrm{a}}$ & $17.72^{\mathrm{a}}$ & $15.90^{\mathrm{a}}$ & $12.96^{\mathrm{a}}$ \\
\hline & \multirow{2}{*}{20} & {$[27]$} & $74.3^{\mathrm{a}}$ & $48.49^{\mathrm{a}}$ & $37.35^{\mathrm{a}}$ & $29.05^{\mathrm{a}}$ & $24.14^{\mathrm{a}}$ & $21.81^{\mathrm{a}}$ & $19.33^{\mathrm{a}}$ & $15.27^{\mathrm{a}}$ \\
\hline & & Present & $73.80^{\mathrm{a}}$ & $48.10^{\mathrm{a}}$ & $37.00^{\mathrm{a}}$ & $28.71^{\mathrm{a}}$ & $23.86^{\mathrm{a}}$ & $21.61^{\mathrm{a}}$ & $19.18^{\mathrm{a}}$ & $15.17^{\mathrm{a}}$ \\
\hline & 50 & [27] & $77.80^{\mathrm{a}}$ & $50.48^{\mathrm{a}}$ & $38.83^{\mathrm{a}}$ & $30.28^{\mathrm{a}}$ & $25.53^{\mathrm{a}}$ & $23.27^{\mathrm{a}}$ & $20.53^{\mathrm{a}}$ & $16.05^{\mathrm{a}}$ \\
\hline & 30 & Present & $77.20^{\mathrm{a}}$ & $50.09^{\mathrm{a}}$ & $38.51^{\mathrm{a}}$ & $30.02^{\mathrm{a}}$ & $25.32^{\mathrm{a}}$ & $23.04^{\mathrm{a}}$ & $20.36^{\mathrm{a}}$ & $15.93^{\mathrm{a}}$ \\
\hline & 100 & [27] & $78.32^{\mathrm{a}}$ & $50.78^{\mathrm{a}}$ & $39.05^{\mathrm{a}}$ & $30.47^{\mathrm{a}}$ & $25.74^{\mathrm{a}}$ & $23.45^{\mathrm{a}}$ & $20.71^{\mathrm{a}}$ & $16.17^{\mathrm{a}}$ \\
\hline & 100 & Present & $77.71^{\mathrm{a}}$ & $50.38^{\mathrm{a}}$ & $38.74^{\mathrm{a}}$ & $30.22^{\mathrm{a}}$ & $25.54^{\mathrm{a}}$ & $23.26^{\mathrm{a}}$ & $20.55^{\mathrm{a}}$ & $16.04^{\mathrm{a}}$ \\
\hline
\end{tabular}

${ }^{a}$ Mode for plate is $(m, n)=(2,1)$.

${ }^{\mathrm{b}}$ Mode for plate is $(m, n)=(3,1)$.

${ }^{c}$ Mode for plate is $(m, n)=(4,1)$.

For convenience, the critical buckling load is presented in nondimensionalized form as

$$
\bar{N}=N_{\mathrm{cr}} \frac{a^{2}}{E_{m} h^{3}} .
$$

Example 1. The first comparison is carried out for simply supported FG plate subjected to uniaxial compression along the $x$-axis $\left(\lambda_{1}=-1, \lambda_{2}=0\right)$. The comparisons of nondimensionalized critical buckling loads $(\bar{N})$ obtained by the present theory and those given by Thai and Choi 
TABLE 2: Comparison of nondimensionalized critical buckling load $(\bar{N})$ of simply supported $\mathrm{Al} / \mathrm{Al}_{2} \mathrm{O}_{3}$ plate subjected to biaxial compression $\left(\lambda_{1}=-1, \lambda_{2}=-1\right)$.

\begin{tabular}{|c|c|c|c|c|c|c|c|c|c|c|}
\hline \multicolumn{11}{|c|}{ Power-law index, $n$} \\
\hline$a / b$ & $a / h$ & Source & 0 & 0.5 & 1 & 2 & 5 & 10 & 20 & 100 \\
\hline \multirow{10}{*}{0.5} & \multirow{2}{*}{5} & {$[27]$} & 5.376 & 3.539 & 2.733 & 2.116 & 1.719 & 1.537 & 1.369 & 1.099 \\
\hline & & Present & 5.371 & 3.527 & 2.715 & 2.092 & 1.700 & 1.527 & 1.364 & 1.097 \\
\hline & \multirow{2}{*}{10} & {$[27]$} & 5.926 & 3.857 & 2.969 & 2.312 & 1.933 & 1.752 & 1.551 & 1.220 \\
\hline & & Present & 5.918 & 3.850 & 2.961 & 2.302 & 1.925 & 1.747 & 1.548 & 1.218 \\
\hline & \multirow{2}{*}{20} & {$[27]$} & 6.079 & 3.857 & 3.034 & 2.367 & 1.996 & 1.815 & 1.604 & 1.255 \\
\hline & & Present & 6.072 & 3.940 & 3.029 & 2.362 & 1.991 & 1.812 & 1.602 & 1.253 \\
\hline & \multirow{2}{*}{50} & {$[27]$} & 6.124 & 3.971 & 3.053 & 2.382 & 2.014 & 1.834 & 1.620 & 1.265 \\
\hline & & Present & 6.117 & 3.966 & 3.049 & 2.379 & 2.011 & 1.831 & 1.618 & 1.263 \\
\hline & \multirow{2}{*}{100} & [27] & 6.131 & 3.974 & 3.056 & 2.385 & 2.016 & 1.837 & 1.622 & 1.266 \\
\hline & & Present & 6.123 & 3.970 & 3.052 & 2.382 & 2.014 & 1.834 & 1.620 & 1.265 \\
\hline \multirow{10}{*}{1} & \multirow{2}{*}{5} & [27] & 8.011 & 5.313 & 4.112 & 3.172 & 2.527 & 2.240 & 2.004 & 1.629 \\
\hline & & Present & 8.001 & 5.288 & 4.073 & 3.120 & 2.487 & 2.221 & 1.994 & 1.626 \\
\hline & \multirow{2}{*}{10} & [27] & 9.289 & 6.062 & 4.670 & 3.632 & 3.018 & 2.726 & 2.417 & 1.910 \\
\hline & & Present & 9.273 & 6.045 & 4.650 & 3.608 & 2.998 & 2.715 & 2.410 & 1.906 \\
\hline & \multirow{2}{*}{20} & [27] & 9.676 & 6.283 & 4.834 & 3.769 & 3.172 & 2.883 & 2.549 & 1.996 \\
\hline & & Present & 9.658 & 6.270 & 4.821 & 3.757 & 3.162 & 2.876 & 2.544 & 1.992 \\
\hline & \multirow{2}{*}{50} & [27] & 9.791 & 6.349 & 4.882 & 3.809 & 3.219 & 2.931 & 2.589 & 2.217 \\
\hline & & Present & 9.772 & 6.336 & 4.872 & 3.801 & 3.212 & 2.925 & 2.584 & 2.018 \\
\hline & \multirow{2}{*}{100} & [27] & 9.807 & 6.358 & 4.889 & 3.815 & 3.225 & 2.938 & 2.595 & 2.025 \\
\hline & & Present & 9.788 & 6.345 & 4.879 & 3.807 & 3.219 & 2.932 & 2.590 & 2.021 \\
\hline \multirow{10}{*}{1.5} & \multirow{2}{*}{5} & [27] & 11.682 & 7.830 & 6.080 & 4.664 & 3.618 & 3.172 & 2.851 & 2.360 \\
\hline & & Present & 11.665 & 7.782 & 6.000 & 4.559 & 3.544 & 3.138 & 2.833 & 2.354 \\
\hline & \multirow{2}{*}{10} & [27] & 14.608 & 9.569 & 7.379 & 5.728 & 4.712 & 4.238 & 3.766 & 2.996 \\
\hline & & Present & 14.571 & 9.528 & 7.331 & 5.671 & 4.666 & 4.212 & 3.749 & 2.987 \\
\hline & \multirow{2}{*}{20} & [27] & 15.589 & 10.133 & 7.798 & 6.076 & 5.101 & 4.630 & 4.096 & 3.214 \\
\hline & & Present & 15.542 & 10.098 & 7.766 & 6.046 & 5.075 & 4.611 & 4.082 & 3.203 \\
\hline & \multirow{2}{*}{50} & {$[27]$} & 15.888 & 10.336 & 7.924 & 6.182 & 5.221 & 4.753 & 4.200 & 3.280 \\
\hline & & Present & 15.837 & 10.270 & 7.897 & 6.160 & 5.203 & 4.737 & 4.186 & 3.270 \\
\hline & \multirow{2}{*}{100} & [27] & 15.931 & 10.328 & 7.942 & 6.197 & 5.239 & 4.771 & 4.215 & 3.290 \\
\hline & & Present & 15.880 & 10.295 & 7.916 & 6.177 & 5.222 & 4.756 & 4.201 & 3.280 \\
\hline \multirow{10}{*}{2} & \multirow{2}{*}{5} & {$[27]$} & 15.724 & 10.662 & 8.309 & 6.335 & 4.775 & 4.138 & 3.739 & 3.153 \\
\hline & & Present & 15.698 & 10.581 & 8.172 & 6.156 & 4.661 & 4.088 & 3.712 & 3.143 \\
\hline & \multirow{2}{*}{10} & {$[27]$} & 21.505 & 14.155 & 10.932 & 8.464 & 6.875 & 6.148 & 5.477 & 4.396 \\
\hline & & Present & 21.429 & 14.071 & 10.830 & 8.345 & 6.782 & 6.095 & 5.444 & 4.378 \\
\hline & \multirow{2}{*}{20} & {$[27]$} & 23.697 & 15.426 & 11.875 & 9.247 & 7.737 & 7.007 & 6.204 & 4.888 \\
\hline & & Present & 23.590 & 15.346 & 11.802 & 9.177 & 7.674 & 6.964 & 6.171 & 4.858 \\
\hline & 50 & [27] & 24.394 & 15.824 & 12.170 & 9.493 & 8.013 & 7.293 & 6.444 & 5.036 \\
\hline & 50 & Present & 24.276 & 15.746 & 12.108 & 9.442 & 7.970 & 7.255 & 6.412 & 5.011 \\
\hline & 100 & [27] & 24.497 & 15.883 & 12.213 & 9.529 & 8.055 & 7.335 & 6.480 & 5.059 \\
\hline & 100 & Present & 24.378 & 15.805 & 12.153 & 9.482 & 8.015 & 7.299 & 6.448 & 5.034 \\
\hline
\end{tabular}

[27] are presented in Table 1. It can be observed that the predicted results are almost identical with that of Thai and Choi [27]. It can also be seen that the critical buckling load decreases with the increase of power-law index value while it increases with the increase of side-to-thickness ratio and aspect ratio. Furthermore, increasing of thickness ratio and aspect ratio not only increases the critical buckling load values, but also causes the changes in critical buckling modes. This can be observed when the plate is subjected to inplane compression along the $x$-axis with aspect ratio value of 2 . The critical buckling mode varies from $(3,1)$ to $(2,1)$ as the side-to-thickness ratio increases from 5 to 10 . The maximum percentage error between present HSDT and Thai and Choi [27] is 3.9 at $a / h=5, a / b=2$, and $n=2$. 
TABLE 3: Comparison of nondimensionalized critical buckling load $(\bar{N})$ of simply supported $\mathrm{Al} / \mathrm{Al}_{2} \mathrm{O}_{3}$ plate subjected to biaxial compression and tension $\left(\lambda_{1}=-1, \lambda_{2}=-1\right)$.

\begin{tabular}{|c|c|c|c|c|c|c|c|c|c|c|}
\hline \multicolumn{11}{|c|}{ Power-law index, $n$} \\
\hline$a / b$ & $a / h$ & Source & 0 & 0.5 & 1 & 2 & 5 & 10 & 20 & 100 \\
\hline \multirow{10}{*}{0.5} & \multirow{2}{*}{5} & [27] & 8.960 & 5.898 & 4.555 & 3.527 & 2.865 & 2.562 & 2.282 & 1.832 \\
\hline & & Present & 8.953 & 5.879 & 4.525 & 3.487 & 2.833 & 2.545 & 2.274 & 1.829 \\
\hline & \multirow{2}{*}{10} & [27] & 9.874 & 6.428 & 4.948 & 3.853 & 3.222 & 2.200 & 2.585 & 2.033 \\
\hline & & Present & 9.863 & 6.416 & 4.934 & 3.837 & 3.208 & 2.911 & 2.580 & 2.031 \\
\hline & \multirow{2}{*}{20} & {$[27]$} & 10.132 & 6.575 & 5.057 & 3.944 & 3.326 & 3.025 & 2.674 & 2.091 \\
\hline & & Present & 10.120 & 6.566 & 5.049 & 3.936 & 3.319 & 3.020 & 2.670 & 2.089 \\
\hline & \multirow{2}{*}{50} & [27] & 10.207 & 6.618 & 5.089 & 3.971 & 3.356 & 3.056 & 2.700 & 2.108 \\
\hline & & Present & 10.195 & 6.610 & 5.082 & 3.965 & 3.352 & 3.052 & 2.697 & 2.105 \\
\hline & \multirow{2}{*}{100} & [27] & 10.218 & 6.624 & 5.093 & 3.974 & 3.361 & 3.061 & 2.704 & 2.110 \\
\hline & & Present & 10.206 & 6.616 & 5.087 & 3.969 & 3.356 & 3.057 & 2.700 & 2.108 \\
\hline \multirow{10}{*}{1} & \multirow{2}{*}{5} & [27] & $26.20^{\mathrm{a}}$ & $17.77^{\mathrm{a}}$ & $13.84^{\mathrm{a}}$ & $10.55^{\mathrm{a}}$ & $7.95^{\mathrm{a}}$ & $6.89^{\mathrm{a}}$ & $6.23^{\mathrm{a}}$ & $5.25^{\mathrm{a}}$ \\
\hline & & Present & $26.16^{\mathrm{a}}$ & $17.63^{\mathrm{a}}$ & $13.62^{\mathrm{a}}$ & $10.26^{\mathrm{a}}$ & $7.76^{\mathrm{a}}$ & $6.81^{\mathrm{a}}$ & $6.18^{\mathrm{a}}$ & $5.23^{\mathrm{a}}$ \\
\hline & \multirow{2}{*}{10} & [27] & $35.84^{\mathrm{a}}$ & $23.59^{\mathrm{b}}$ & $18.22^{\mathrm{a}}$ & $14.10^{\mathrm{a}}$ & $11.45^{\mathrm{a}}$ & $10.24^{\mathrm{a}}$ & $9.12^{\mathrm{a}}$ & $7.32^{\mathrm{a}}$ \\
\hline & & Present & $35.71^{\mathrm{a}}$ & $23.45^{\mathrm{b}}$ & $18.04^{\mathrm{a}}$ & $13.90^{\mathrm{a}}$ & $11.30^{\mathrm{a}}$ & $10.15^{\mathrm{a}}$ & $9.07^{\mathrm{a}}$ & 7.297 \\
\hline & \multirow{2}{*}{20} & [27] & $39.49^{\mathrm{a}}$ & $25.71^{\mathrm{a}}$ & $19.79^{\mathrm{a}}$ & $15.41^{\mathrm{a}}$ & $12.88^{\mathrm{a}}$ & $11.67^{\mathrm{a}}$ & $10.34^{\mathrm{a}}$ & $8.13^{\mathrm{a}}$ \\
\hline & & Present & $39.31^{\mathrm{a}}$ & $25.57^{\mathrm{a}}$ & $19.67^{\mathrm{a}}$ & $15.29^{\mathrm{a}}$ & $12.79^{\mathrm{a}}$ & $11.60^{\mathrm{a}}$ & $10.28^{\mathrm{a}}$ & $8.09^{\mathrm{a}}$ \\
\hline & \multirow{2}{*}{50} & [27] & $40.65^{\mathrm{a}}$ & $26.37^{\mathrm{a}}$ & 20.283 & $15.82^{\mathrm{a}}$ & $13.35^{\mathrm{a}}$ & $12.15^{\mathrm{a}}$ & $10.74^{\mathrm{a}}$ & $8.39^{\mathrm{b}}$ \\
\hline & & Present & $40.46^{\mathrm{a}}$ & $26.24^{\mathrm{a}}$ & 20.179 & $15.73^{\mathrm{a}}$ & $13.28^{\mathrm{a}}$ & $12.09^{\mathrm{a}}$ & $10.68^{\mathrm{a}}$ & $8.35^{\mathrm{b}}$ \\
\hline & \multirow{2}{*}{100} & [27] & $40.82^{\mathrm{a}}$ & $26.47^{\mathrm{a}}$ & $20.35^{\mathrm{a}}$ & $15.88^{\mathrm{a}}$ & $13.42^{\mathrm{a}}$ & $12.22^{\mathrm{a}}$ & $10.79^{\mathrm{a}}$ & $8.43^{\mathrm{b}}$ \\
\hline & & Present & $40.62^{\mathrm{a}}$ & $26.34^{\mathrm{a}}$ & $20.25^{\mathrm{a}}$ & $15.80^{\mathrm{a}}$ & $13.35^{\mathrm{a}}$ & $12.16^{\mathrm{a}}$ & $10.74^{\mathrm{a}}$ & $8.39^{\mathrm{b}}$ \\
\hline \multirow{10}{*}{1.5} & \multirow{2}{*}{5} & [27] & $29.02^{b}$ & $20.11^{\mathrm{b}}$ & $15.78^{\mathrm{b}}$ & $11.90^{\mathrm{b}}$ & $8.52^{\mathrm{b}}$ & $7.24^{\mathrm{b}}$ & $6.60^{\mathrm{b}}$ & $5.74^{\mathrm{b}}$ \\
\hline & & Present & $28.97^{\mathrm{b}}$ & $19.92^{\mathrm{b}}$ & $15.45^{\mathrm{b}}$ & $11.47^{\mathrm{b}}$ & $8.29^{\mathrm{b}}$ & $7.15^{\mathrm{b}}$ & $6.54^{\mathrm{b}}$ & $5.72^{\mathrm{b}}$ \\
\hline & \multirow{2}{*}{10} & [27] & 37.982 & 24.878 & 19.186 & 14.893 & 12.252 & 11.020 & 9.791 & 7.789 \\
\hline & & Present & 37.884 & 24.773 & 19.060 & 14.745 & 12.133 & 10.950 & 9.748 & 7.767 \\
\hline & \multirow{2}{*}{20} & [27] & 40.531 & 26.346 & 20.244 & 15.798 & 13.262 & 12.038 & 10.650 & 8.355 \\
\hline & & Present & 40.408 & 26.255 & 20.190 & 15.718 & 13.194 & 11.988 & 10.612 & 8.329 \\
\hline & \multirow{2}{*}{50} & [27] & 41.308 & 26.789 & 20.601 & 16.072 & 13.575 & 12.358 & 10.919 & 8.529 \\
\hline & & Present & 41.177 & 26.702 & 20.532 & 16.016 & 13.528 & 12.317 & 10.883 & 8.502 \\
\hline & \multirow{2}{*}{100} & [27] & 41.421 & 26.854 & 20.649 & 16.112 & 13.624 & 12.405 & 10.958 & 8.554 \\
\hline & & Present & 41.289 & 26.768 & 20.582 & 16.059 & 13.577 & 12.365 & 10.923 & 8.527 \\
\hline \multirow{10}{*}{2} & \multirow{2}{*}{5} & [27] & 26.206 & 17.770 & 13.849 & 10.559 & 7.959 & 6.897 & 6.232 & 5.256 \\
\hline & & Present & 26.164 & 17.636 & 13.620 & 10.261 & 7.768 & 6.814 & 6.187 & 5.239 \\
\hline & \multirow{2}{*}{10} & [27] & 35.842 & 23.592 & 18.221 & 14.107 & 11.458 & 10.247 & 9.128 & 7.326 \\
\hline & & Present & 35.715 & 23.451 & 18.050 & 13.909 & 11.303 & 10.159 & 9.073 & 7.297 \\
\hline & \multirow{2}{*}{20} & [27] & 39.495 & 25.710 & 19.793 & 15.412 & 12.888 & 11.678 & 10.340 & 8.134 \\
\hline & & Present & 39.317 & 25.576 & 19.670 & 15.295 & 12.791 & 11.607 & 10.286 & 8.096 \\
\hline & 50 & [27] & 40.657 & 26.374 & 20.283 & 15.822 & 13.355 & 12.154 & 10.740 & 8.393 \\
\hline & 0 & Present & 40.460 & 26.243 & 20.179 & 15.737 & 13.284 & 12.092 & 10.687 & 8.352 \\
\hline & 100 & [27] & 40.829 & 26.472 & 20.355 & 15.882 & 13.425 & 12.226 & 10.800 & 8.432 \\
\hline & & Present & 40.629 & 26.341 & 20.254 & 15.803 & 13.358 & 12.165 & 10.747 & 8.390 \\
\hline
\end{tabular}

${ }^{\mathrm{a}}$ Mode for plate is $(m, n)=(2,1)$.

${ }^{\mathrm{b}}$ Mode for plate is $(m, n)=(1,2)$.

Example 2. The next comparison is performed for the simply supported FG plate subjected to inplane biaxial compression $\left(\lambda_{1}=-1, \lambda_{2}=-1\right)$. The results of critical buckling loads obtained by the present theory and those reported by Thai and Choi [27] are presented in Table 2 and observed the close agreement between the results. It can be seen that, in this loading condition also, the nondimensionalized critical buckling load decreases with the increase of power-law index, while it increases with the increase of aspect ratio and sideto-thickness ratio, but only one critical buckling mode exists 
TABLE 4: Comparison of nondimensionalized critical buckling load $(\bar{N})$ of simply supported $\mathrm{Al} / \mathrm{Al}_{2} \mathrm{O}_{3}$ plate subjected to uniaxial compression $\left(\lambda_{1}=-1, \lambda_{2}=0\right)$.

\begin{tabular}{|c|c|c|c|c|c|c|c|c|c|}
\hline \multirow{2}{*}{$b / h$} & \multicolumn{3}{|c|}{$n=0.1$} & \multicolumn{3}{|c|}{$n=1$} & \multicolumn{3}{|c|}{$n=10$} \\
\hline & CPT [16] & FSDT [16] & HSDT & СРТ [16] & FSDT [16] & HSDT & СРТ [16] & FSDT [16] & HSDT \\
\hline 10 & 17.68 & 16.76 & 16.74 & 9.78 & 9.33 & 9.29 & 5.87 & 5.66 & 5.42 \\
\hline 20 & 17.68 & 17.4 & 17.41 & 9.78 & 9.66 & 9.64 & 5.87 & 5.78 & 5.75 \\
\hline 40 & 17.68 & 17.62 & 17.59 & 9.78 & 9.75 & 9.73 & 5.87 & 5.85 & 5.83 \\
\hline 50 & 17.68 & 17.64 & 17.61 & 9.78 & 9.76 & 9.74 & 5.87 & 5.86 & 5.84 \\
\hline 100 & 17.68 & 17.67 & 17.64 & 9.78 & 9.77 & 9.75 & 5.87 & 5.87 & 5.86 \\
\hline
\end{tabular}

in any case of the aspect ratio, thickness ratio, modulus ratio, and power-law index. The maximum percentage error between present HSDT and Thai and Choi [27] is 2.90 at $a / h=5, a / b=2$, and $n=2$.

Example 3. The third comparison is carried out for the simply supported FG plates under inplane biaxial compression and tension $\left(\lambda_{1}=-1, \lambda_{2}=1\right)$. The results predicted by present theory are compared with Thai and Chois [27] results and good agreement between the results can be observed. The results are presented in Table 3 . It can also be seen that, under biaxial compression and tension, the critical buckling load decreases with the increase of power-law index value while it increases with the increase of side-to-thickness ratio and aspect ratio, same as in uniaxial and biaxial compression. Also, increasing of thickness ratio and aspect ratio not only increases the critical buckling load values, but also causes changes in critical buckling modes. This can be observed when the aspect ratio value is 1.5. The critical buckling mode varies from $(2,1)$ to $(1,2)$ for the values and the side-tothickness ratio increases from 5 to 10 and 20 to 50 . The maximum percentage error between present HSDT and Thai and Choi [27] is 2.90 at $a / h=5, a / b=2$, and $n=2$.

Example 4. The last comparison is carried out for the simply supported FG plates under inplane uniaxial compression $\left(\lambda_{1}=-1, \lambda_{2}=0\right)$. The results predicted by present theory are compared with the classical plate theory (CPT) [16] and firstorder shear deformation theory (FSDT) [16] are presented in Table 4. The maximum percentage error between present HSDT and CPT and HSDT and FSDT for $n=0.1,1$, and 10 , respectively are $5.633954,0.19667,5.195466$, and 0.42725 , $8.294579,4.39547$ at $a / h=10$. It is also seen that, the CPT and FSDT overpredicts the critical buckling load for all thickness ratios.

4.2. Parametric Study. The effect of side-to-thickness ratio, aspect ratio, and the modulus ratio on nondimensionalized critical buckling load for simply supported FG plate made of $\mathrm{Al} / \mathrm{Al}_{2} \mathrm{O}_{3}$ with $\varepsilon_{z}=0$ is investigated. Figures $2-4$ represent the variation of nondimensionalized critical buckling load with side-to-thickness ratio, aspect ratio, and modulus ratio, respectively, under uniaxial compression. From Figures 2-4, it is important to observe that the nondimensionalized critical buckling loads are higher for ceramic rich plates and lower for metal rich plates. The critical buckling loads of FGM plates

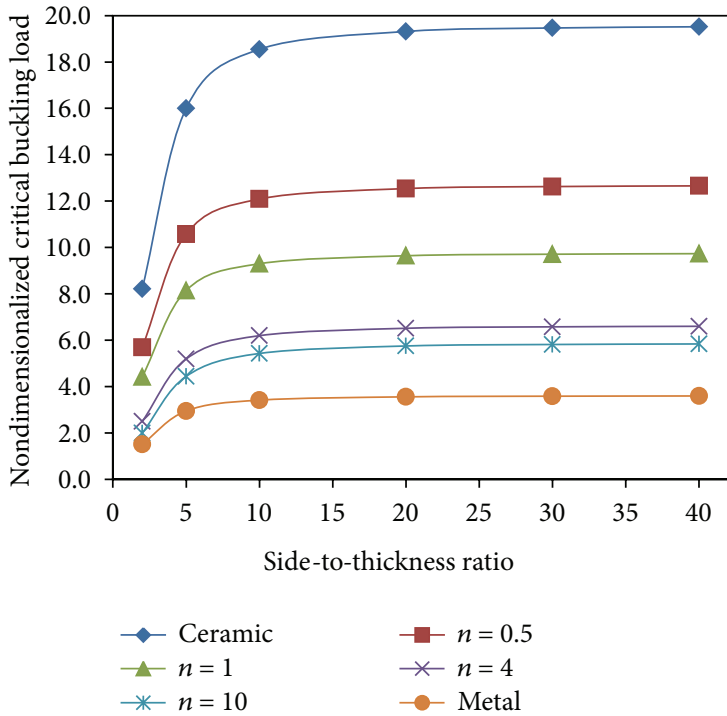

FIGURE 2: Effect of side-to-thickness ratios $(a / h)$ on nondimensionalized critical buckling load $(\bar{N})$ under uniaxial compression for a simply supported FG plate for various material variation parameters $(n)$.

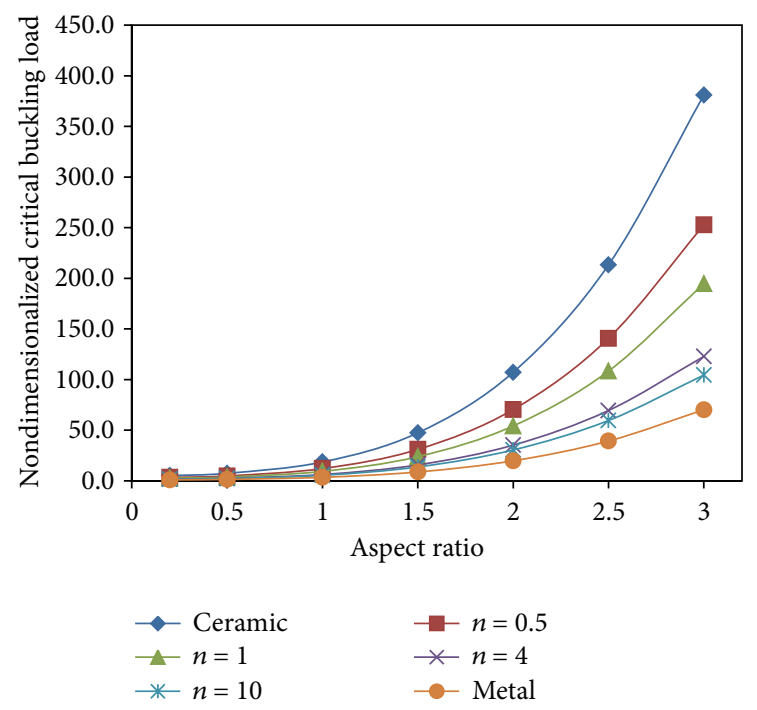

FIGURE 3: Effect of aspect ratios $(a / b)$ on nondimensionalized critical buckling load $(\bar{N})$ under uniaxial compression for a simply supported FG plate for various material variation parameters $(n)$. 


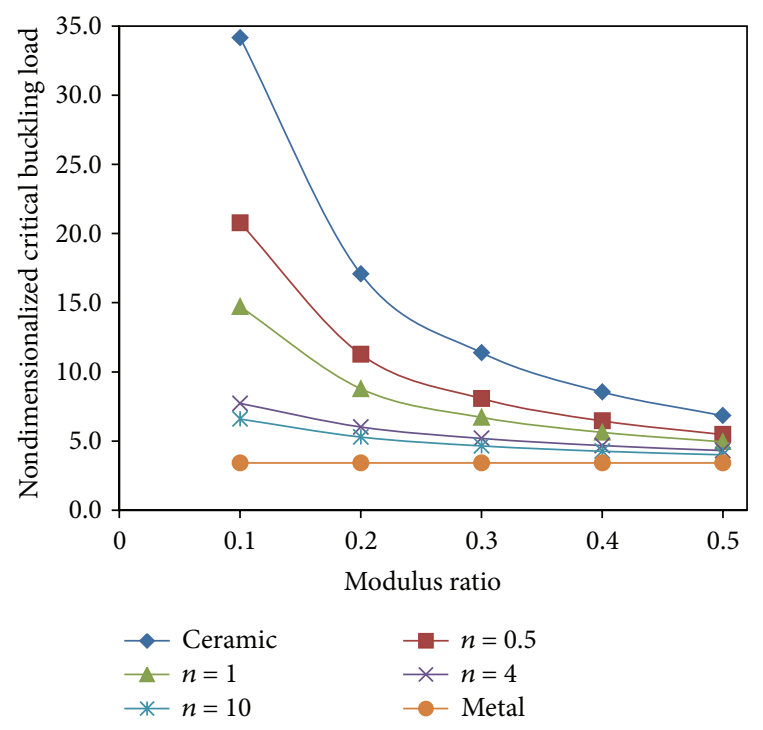

FIgURE 4: Effect of modulus ratio $\left(E_{m} / E_{c}\right)$ on nondimensionalized critical buckling load $(\bar{N})$ under uniaxial compression for a simply supported FG plate for various material variation parameters $(n)$.

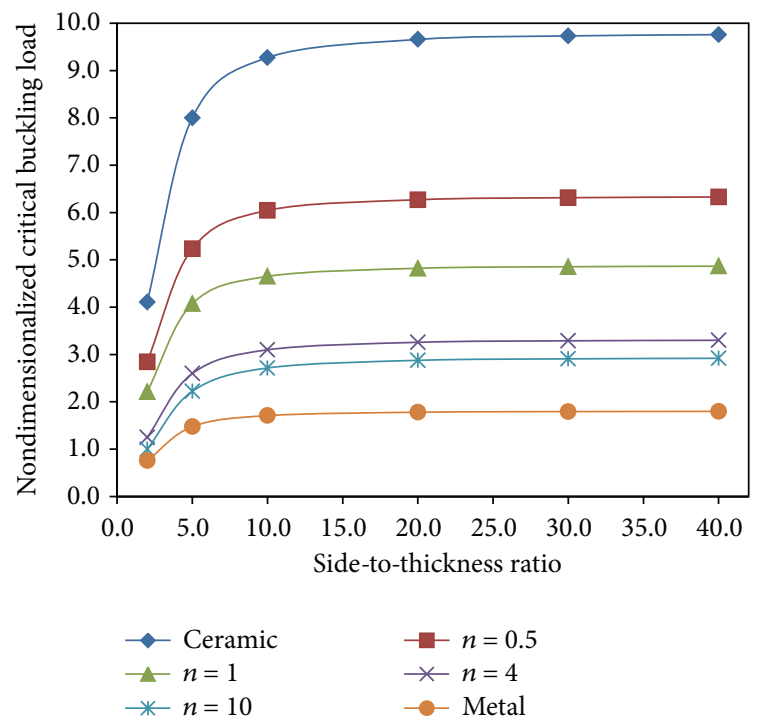

FIGURE 5: Effect of side to thickness ratios $(a / h)$ on nondimensionalized critical buckling load $(\bar{N})$ under biaxial compression for a simply supported FG plate for various material variation parameters $(n)$.

are intermediate to that of ceramic and metal. From Figure 2 it is seen that the effect of shear deformation is significant for a side-to-thickness ratio less than 10 and diminishes with increase of side-to-thickness ratio. The increase of aspect ratio increases the critical buckling load due to increase of stiffness of the plate as shown in Figure 3.

From Figure 4, it can be seen that the nondimensional critical buckling load decreases as the metal-to-ceramic modulus ratio increases and decreases as the power law index increases. This is due to the fact that higher values of metal-toceramic modulus ratio correspond to high portion of metal.

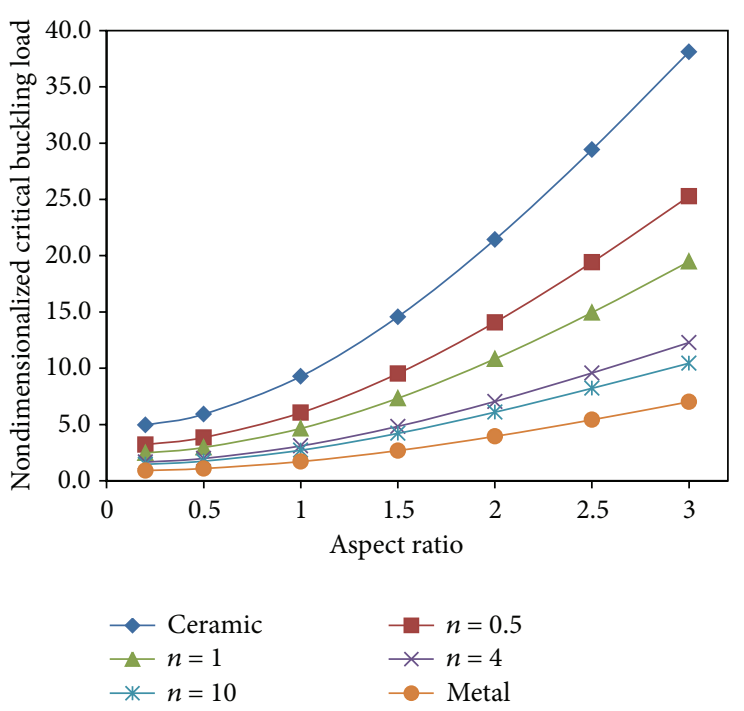

FIGURE 6: Effect of aspect ratios $(a / b)$ on nondimensionalized critical buckling load $(\bar{N})$ under biaxial compression for a simply supported FG plate for various material variation parameters $(n)$.

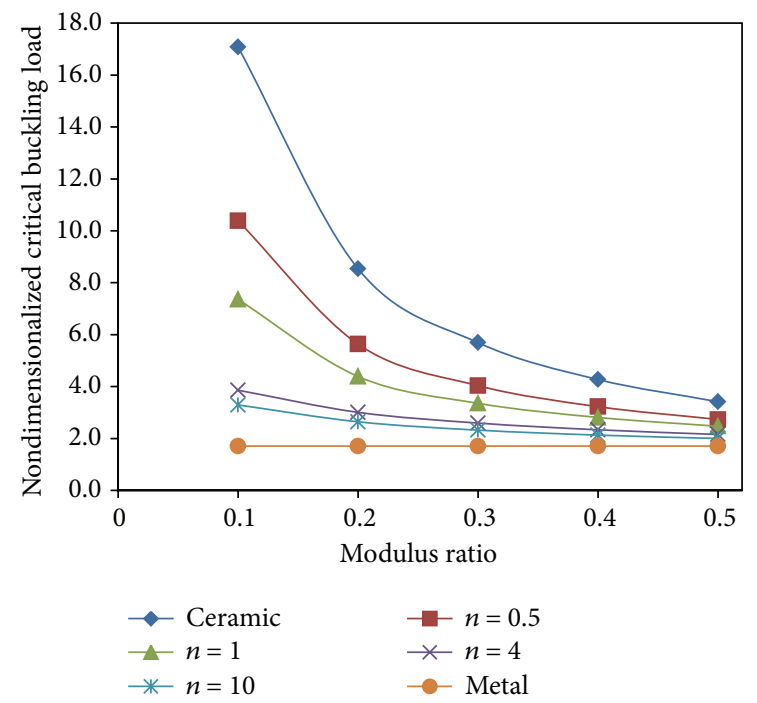

FIGURE 7: Effect of modulus ratios $\left(E_{m} / E_{c}\right)$ on nondimensionalized critical buckling load $(\bar{N})$ under biaxial compression for a simply supported FG plate for various material variation parameters $(n)$.

The effect of side-to-thickness ratio, aspect ratio, modulus ratio, and power law index values on nondiensionalized critical buckling load for a simply supported FG plate under biaxial compression is shown in Figures 5, 6, and 7. The same can be observed as in the case of uniaxial compression. It is important to observe that the critical buckling loads are larger in uniaxial compression and smaller in biaxial compression. Figure 8 shows the variation of nondimensionalized critical buckling load of different modulus ratios and power law index values under inplane compression and tension. It can be observed that critical buckling load decreases with the increase of modulus ratio and power-law index values. 


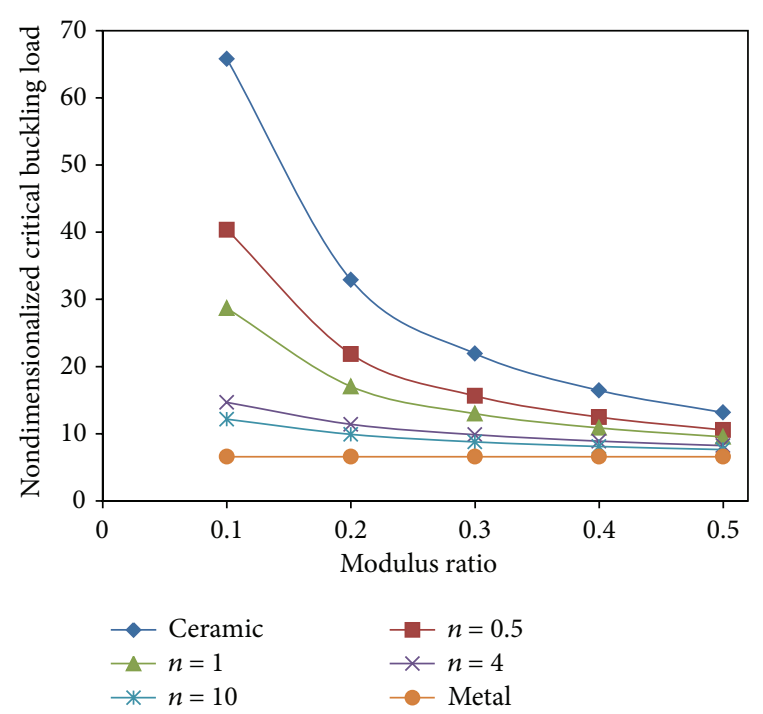

FIGURE 8: Effect of modulus ratios $\left(E_{m} / E_{c}\right)$ on nondimensionalized critical buckling load $(\bar{N})$ under biaxial compression and tension for a simply supported FG plate for various material variation parameters $(n)$ (mode for the plate $(m, n)=(1,2))$.

\section{Conclusions}

A higher order shear deformation theory was successfully developed and applied to study the buckling behavior of functionally graded plates without enforcing zero transverse shear stresses on the top and bottom surfaces of the plate. The present formulation was compared with the refined theory developed by Thai and Choi [27] and proved very accurate for the buckling problem. This eliminated the need of using shear correction factors. It can be concluded that the present theory is accurate and efficient in predicting the critical buckling load of simply supported FG plates. Hence, the present findings will be useful benchmark for evaluating other future plate theories and numerical methods such as the finite element and meshless methods.

\section{References}

[1] R. Javaheri and M. R. Eslami, "Buckling of functionally graded plates under in-plane compressive loading," Journal of Applied Mathematics and Mechanics, vol. 82, no. 4, pp. 277-283, 2002.

[2] S. Abrate, "Functionally graded plates behave like homogeneous plates," Composites B, vol. 39, no. 1, pp. 151-158, 2008.

[3] M. Mohammadi, A. R. Saidi, and E. Jomehzadeh, "Levy solution for buckling analysis of functionally graded rectangular plates," Applied Composite Materials, vol. 17, no. 2, pp. 81-93, 2010.

[4] M. Mahdavian, "Buckling analysis of simply-supported functionally graded rectangular plates under non-uniform in-plane compressive loading," Journal of Solid Mechanics, vol. 1, no. 3, pp. 213-225, 2009.

[5] E. Feldman and J. Aboudi, "Buckling analysis of functionally graded plates subjected to uniaxial loading," Composite Structures, vol. 38, no. 1-4, pp. 29-36, 1997.
[6] B. A. S. Shariat, R. Javaheri, and M. R. Eslami, "Buckling of imperfect functionally graded plates under in-plane compressive loading," Thin-Walled Structures, vol. 43, no. 7, pp. 10201036, 2005.

[7] H. V. Tung and N. D. Duc, "Nonlinear analysis of stability for functionally graded plates under mechanical and thermal loads," Composite Structures, vol. 92, no. 5, pp. 1184-1191, 2010.

[8] E. Reissner, "The effect of transverse shear deformation on the bending of elastic plates," ASME Journal of Applied Mechanics, vol. 12, no. 2, pp. 69-77, 1945.

[9] R. D. Mindlin, "Influence of rotary inertia and shear on flexural motions of isotropic, elastic plates," ASME Journal of Applied Mechanics, vol. 18, pp. 31-38, 1951.

[10] H. T. Thai and T. P. Vo, "A new sinusoidal shear deformation theory for bending, buckling, and vibration of functionally graded plates," Applied Mathematical Modelling, vol. 37, no. 5, pp. 3269-3281, 2013.

[11] J. Yang, K. M. Liew, and S. Kitipornchai, "Second-order statistics of the elastic buckling of functionally graded rectangular plates," Composites Science and Technology, vol. 65, no. 7-8, pp. 11651175, 2005.

[12] M. Mohammadi, A. R. Saidi, and E. Jomehzadeh, "A novel analytical approach for the buckling analysis of moderately thick functionally graded rectangular plates with two simplysupported opposite edges," Proceedings of the Institution of Mechanical Engineers C, vol. 224, no. 9, pp. 1831-1841, 2010.

[13] X. Zhao, Y. Y. Lee, and K. M. Liew, "Mechanical and thermal buckling analysis of functionally graded plates," Composite Structures, vol. 90, no. 2, pp. 161-171, 2009.

[14] H. A. Sepiani, A. Rastgoo, F. Ebrahimi, and A. G. Arani, "Vibration and buckling analysis of two-layered functionally graded cylindrical shell, considering the effects of transverse shear and rotary inertia," Materials and Design, vol. 31, no. 3, pp. 1063-1069, 2010.

[15] A. Naderi and A. R. Saidi, "On pre-buckling configuration of functionally graded Mindlin rectangular plates," Mechanics Research Communications, vol. 37, no. 6, pp. 535-538, 2010.

[16] R. Saha and P. R. Maiti, "Buckling of simply supported FGM plates under uniaxial load," International Journal of Civil and Structural Engineering, vol. 2, no. 4, pp. 1036-1050, 2012.

[17] R. Javaheri and M. R. Eslami, "Thermal buckling of functionally graded plates based on higher order theory," Journal of Thermal Stresses, vol. 25, no. 7, pp. 603-625, 2002.

[18] M. M. Najafizadeh and H. R. Heydari, "Thermal buckling of functionally graded circular plates based on higher order shear deformation plate theory," European Journal of Mechanics A, vol. 23, no. 6, pp. 1085-1100, 2004.

[19] M. Bodaghi and A. R. Saidi, "Levy-type solution for buckling analysis of thick functionally graded rectangular plates based on the higher-order shear deformation plate theory," Applied Mathematical Modelling, vol. 34, no. 11, pp. 3659-3673, 2010.

[20] E. Bagherizadeh, Y. Kiani, and M. R. Eslami, "Mechanical buckling of functionally graded material cylindrical shells surrounded by Pasternak elastic foundation," Composite Structures, vol. 93, no. 11, pp. 3063-3071, 2011.

[21] H. Mozafari and A. Ayob, "Effect of thickness variation on the mechanical buckling load in plates made of functionally graded materials," Procedia Technology, vol. 1, pp. 496-504, 2012.

[22] L. S. Ma and T. J. Wang, "Nonlinear bending and post-buckling of a functionally graded circular plate under mechanical and thermal loadings," International Journal of Solids and Structures, vol. 40, no. 13-14, pp. 3311-3330, 2003. 
[23] S. Hosseini-Hashemi, K. Khorshidi, and M. Amabili, "Exact solution for linear buckling of rectangular Mindlin plates," Journal of Sound and Vibration, vol. 315, no. 1-2, pp. 318-342, 2008.

[24] A. R. Saidi, A. Rasouli, and S. Sahraee, "Axisymmetric bending and buckling analysis of thick functionally graded circular plates using unconstrained third-order shear deformation plate theory," Composite Structures, vol. 89, no. 1, pp. 110-119, 2009.

[25] O. O. Oyekoya, D. U. Mba, and A. M. El-Zafrany, "Buckling and vibration analysis of functionally graded composite structures using the finite element method," Composite Structures, vol. 89, no. 1, pp. 134-142, 2009.

[26] S. A. M. Ghannadpour, H. R. Ovesy, and M. Nassirnia, "Buckling analysis of functionally graded plates under thermal loadings using the finite strip method," Computers and Structures, vol. 108-109, pp. 93-99, 2012.

[27] H. T. Thai and D. H. Choi, "An efficient and simple refined theory for buckling analysis of functionally graded plates," Applied Mathematical Modelling, vol. 36, no. 3, pp. 1008-1022, 2012.

[28] B. Uymaz and M. Aydogdu, "Three dimensional mechanical buckling of FG plates with general boundary conditions," Composite Structures, vol. 96, pp. 174-193, 2013.

[29] A. Lal, K. R. Jagtap, and B. N. Singh, "Post buckling response of functionally graded materials plate subjected to mechanical and thermal loadings with random material properties," Applied Mathematical Modelling, vol. 37, no. 5, pp. 2900-2920, 2013. 

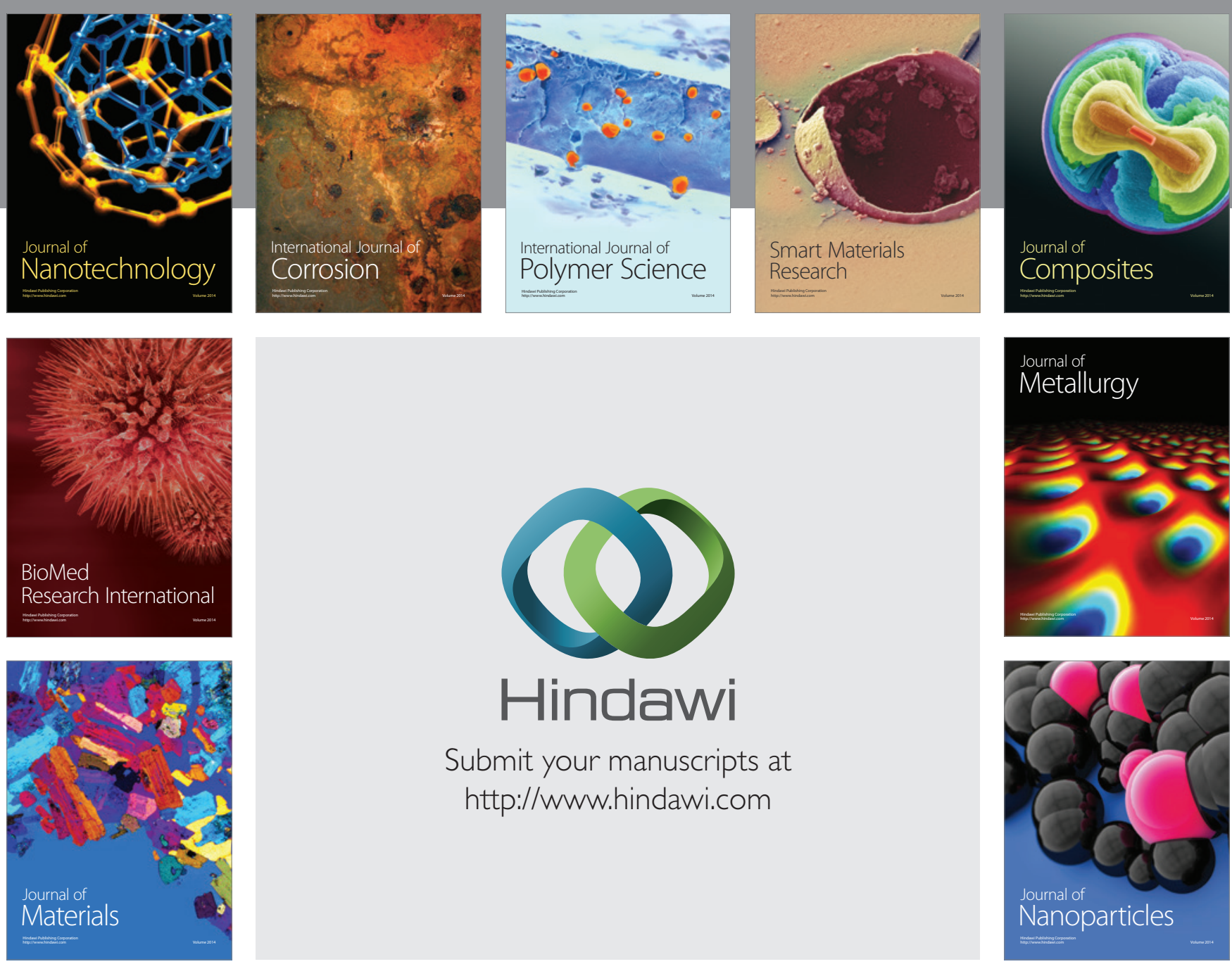

Submit your manuscripts at http://www.hindawi.com
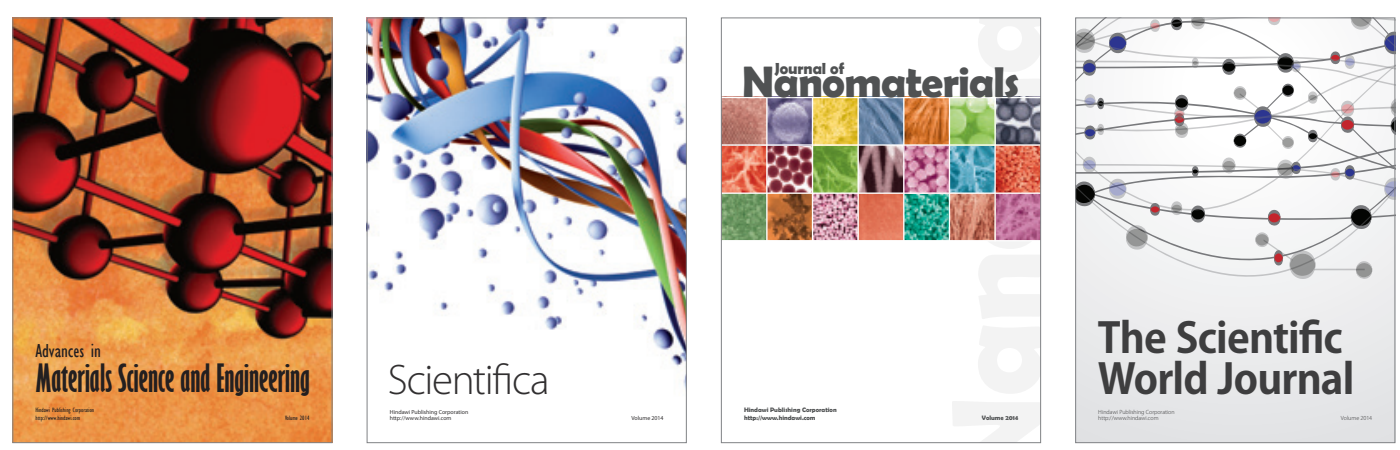

\section{The Scientific World Journal}
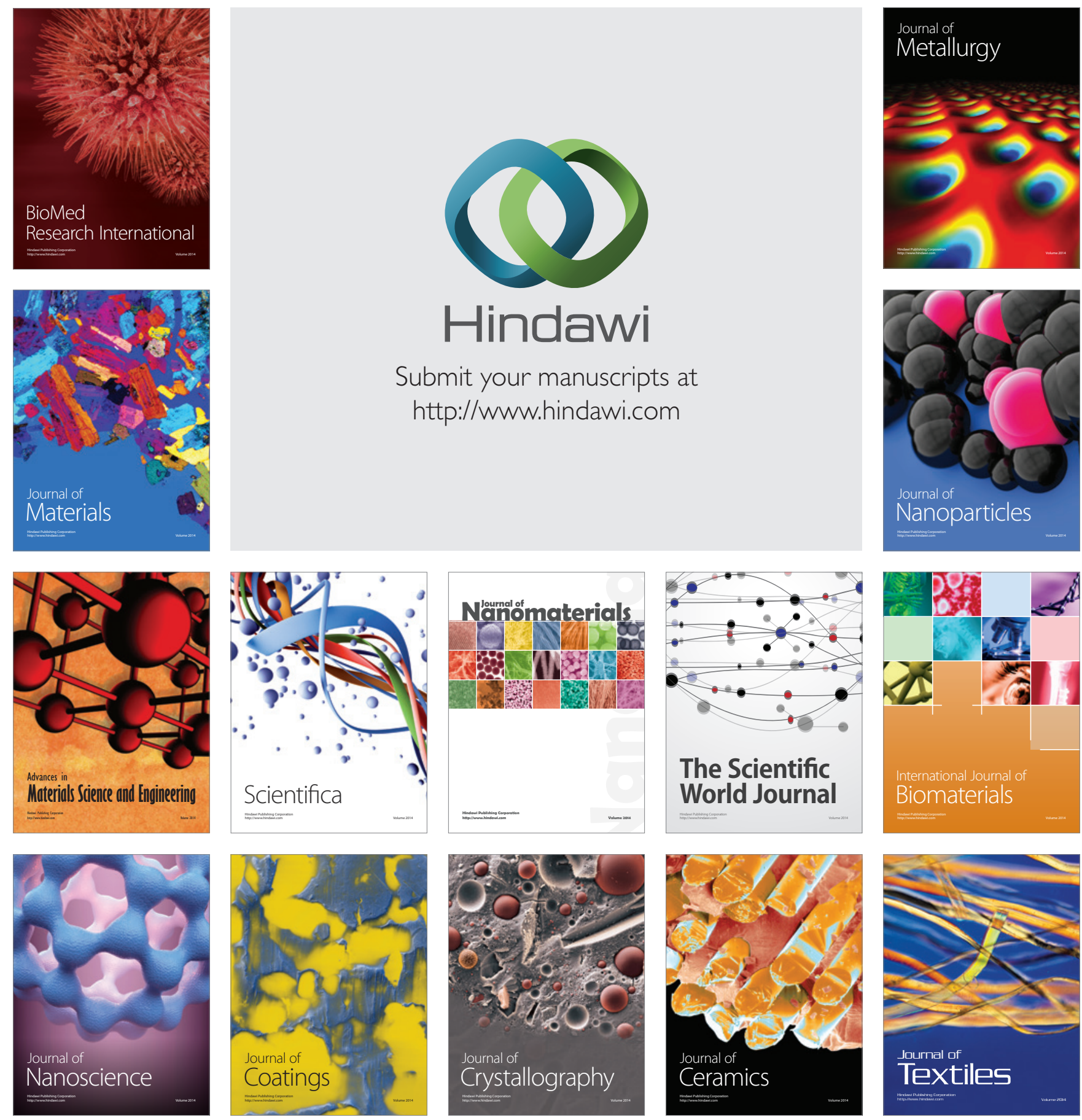